\title{
Web Services Composition: A Story of Models, Automata, and Logics
}

\author{
Richard Hull \\ Director of Network Data and Services Research \\ Bell Labs Research, Lucent Technologies \\ hull@1ucent.com
}

The web services paradigm, which finds roots in Service-Oriented Computing [1, 12], promises to enable rich, flexible, and dynamic interoperation of highly distributed and heterogeneous web-hosted services. Substantial progress has already been made towards this goal (e.g., emerging standards such as SOAP, WSDL, BPEL) and industrial technology (e.g., IBM's WebSphere Toolkit, Sun's Open Net Environment and JiniTM Network technology, Microsoft's .Net and Novell's One Net initiatives, HP's e-speak). Several research efforts are already underway that build on or take advantage of the paradigm, including the DAMLS/OWL-S program [9, 10, 7, 13], the Semantic Web Services Initiative (SWSI) [14] andWeb Service Modeling Ontology (WSMO) [16] groups, and automata-based models for web services [5, 8, 4].

A key research challenge in web services concerns (semi-)automatic discovery and composition of web services, in order to construct new web services with desired properties or capabilities. This talk provides a survey of key developments that work towards this ambitious goal.

The fundamental work in this area has centered on three models, each coming with a different approach to the composition problem. The OWL-S model for web services focuses on how web services interact with the "real world", represented abstractly using (time-varying) first-order logic predicates and terms. A representative composition result [11] here uses a translation into Petri nets. The "Roman" model for services [4] focuses on an abstract notion of "activities" (without modeling how they impact the world), and in essence model web services as finite state automata with transitions labeled by these activities. A powerful composition result is obtained using a reduction to Propositional Dynamic Logic (PDL). The Conversation model [5] focuses on messages passed between web services, and again uses finite state automata to model the internal processing of a service, with transitions labeled by message sends and message reads. A key result here concerns determination of the "local" behavior of individual services, if they are required to conform to a given "global" behavior (as might be specified using choreography constraints, reminiscent of WS-Choreography [17]).

The talk also discusses two on-going efforts to unify the three models just described. One activity, by the Semantic Web Services Intiative (SWSI), is to develop a Semantic Web Services Ontology (SWSO) (see [15]). This is based on the Process Specification Language (PSL) [6], a first-order ontology for sharing descriptions of manufacturing processes, which recently became an ISO standard.

The first-order logic embodiment of SWSO extends PSL with key web service constructs, including (a) OWL-S style atomic services that can impact the "real world", (b) messages, which provide an abstraction of standards such as WSDL and WS-Choreography, and (c) various ways to model the process and data flow inside services. A second activity is embodied in the Colombo model of web services, which combines key aspects of OWL-S, of the Roman model, and of the Conversation model. References $[2,3]$ obtain a first composition result in this richer model, that generalizes the results obtained earlier for the Roman model. 


\section{References}

[1] G. Alonso, F. Casati, H. Kuno, and V. Machiraju. Web Services. Concepts, Architectures and Applications. Springer, 2004.

[2] D. Berardi, D. Calvanese, G. De Giacomo, R. Hull, and M. Mecella. Automatic composition of web services in Colombo. In Proc. of 13th Itallian Symp. on Advanced Database Systems, June 2005.

[3] D. Berardi, D. Calvanese, G. De Giacomo, R. Hull, and M. Mecella. Automatic Composition of Transition-based Semantic Web Services with Messaging. Technical report, Dipartimento di Informatica e Sistemistica, Universit’a di Roma "La Sapienza", Roma, Italy, April, 2005.

[4] D. Berardi, D. Calvanese, G. De Giacomo, M. Lenzerini, and M. Mecella. Automatic Composition of $e$-Services that Export their Behavior. In Proceedings of the 1st InternationalConference on Service Oriented Computing (ICSOC 2003), volume 2910 of LNCS, pages 43-58. Springer, 2003.

[5] T. Bultan, X. Fu, R. Hull, and J. Su. Conversation Specification: A New Approach to Design and Analysis of E-Service Composition. In Proceedings of the 12th International World Wide Web Conference (WWW 2003), pages 403-410. ACM, 2003.

[6] M. Gruninger. A Guide to the Ontology of the Process Specification Language. In R. Studer and S. Staab, editors, Handbook of Ontologies, pages 575-592. Springer-Verlag, 2003.

[7] M. Gr"uninger. Applications of PSL to semantic web services. In Proceedings of SWDB'03, The first International Workshop on Semantic Web and Databases, co-located with VLDB '03, 2003.

[8] R. Hull, M. Benedikt, V. Christophides, and J. Su. EServices: A Look Behind the Curtain. In Proceedings of the 22nd ACM SIGACT SIGMOD SIGART Symposium on Principles of Database Systems (PODS 2003), pages 1-14. ACM, 2003.

[9] D. Martin, M. Paolucci, S. McIlraith, M. Burstein, D. Mc-Dermott, D. McGuinness, B. Parsia, T. Payne, M. Sabou, M. Solanki, N. Srinivasan, and K. Sycara. Bringing Semantics to Web Services: The OWL-S Approach. In 1st International Workshop on Semantic Web Services and Web Process Composition (SWSWPC 2004), 2004.

[10] S. McIlraith, T.C. Son, and H. Zeng. SemanticWeb Services. IEEE Intelligent Systems, 16(2):46 - 53, 2001.

[11] S. Narayanan and S. McIlraith. Simulation, Verification and Automated Composition of Web Services. In Proceedings of the 11th International World Wide Web Conference (WWW 2002), pages 77 - 88. ACM Press, 2002.

[12] M.P. Papazoglou and D. Georgakopoulos. Service Oriented Computing (special issue). Communication of the ACM, 46(10):24-28, 2003.

[13] Katia P. Sycara, Massimo Paolucci, Julien Soudry, and Naveen Srinivasan. Dynamic discovery and coordination of agent-based semantic web services. IEEE Internet Computing, 8(3):66-73, 2004.

[14] Semantic Web Services Initiative working group. SWSI home page (http: //www . swsi .org/).

[15] SWSL Committee. Semantic web service ontology (SWSO), 2005. Available in http://www.daml.org/services/swsl/report/.

[16] Web Service Modeling Ontology working group. WSMO home page (http: / /www. wsmo.org/).

[17] WS-Choreography working group. WS-choreography home page (http: / / www . w3 . org/2002/ws/chor/).

\section{About the speaker}

Richard Hull is Director of Network Data and Services Research at Bell Laboratories, a division of Lucent Technologies. Hull has broad research interests in the areas of data and information management. He has published over 75 journal and conference articles, and is co-author of the book "Foundations of Databases" (Addison-Wesley). Hull has served on or chaired numerous conference program committees, and is now Associate Editor of the ACM Transactions on Database Systems journal.

Hull received a Ph.D. degree in Mathematics from the University of California, Berkeley, in 1979. He spent many years on the faculty of Computer Science at the University of Southern California, and has spent numerous summers at INRIA in France. During that period Hull performed research on database theory, database models, and database programming languages, and his research was supported in part by grants from NSF, DARPA, AT\&T, and U S WEST. Since joining Bell Labs, Hull's research has focused on e-services, pervasive computing, personalization, and data management. Hull has also been instrumental in the development of Lucent products in the areas of customer care, privacy-conscious personalization, and more broadly, for enabling the next generation of converged services that span telecom and the web. 